\title{
Modélisation diphasique du transport de sédiments cohésifs
}

\author{
Catherine Villaret, Charles Teisson, Olivier Simonin \\ Ingénieurs de recherche, Laboratoire National d'Hydraulique, Chatou
}

Cosette Boeuf

Etudiante en D.E.A. de Mécanique, Université de la Méditerranée, Marseille

\section{Résumé}

Un modèle diphasique de transport sédimentaire a été développé au LNH dans le cas d'écoulement uniforme (modèle $1 \mathrm{D}$ vertical). Ce modèle a été tout d'abord validé pour le transport en suspension de sédiments non-cohésifs et permet de redroduire de manière satisfaisante des mesures de profils des vitesses et de concentration. Le modèle a été ensuite adapté pour prendre en compte les mécanismes de floculation. A partir d'un état défloculé, on observe la croissance des agrégats jusqu'à une valeur limite. La prise en compte du changement de densité des agrégats, par un terme d'échange de masse entre les phases liquide et solide, permet d'accélérer le processus de floculation.

\section{Introduction}

La modélisation du transport sédimentaire est un sujet d'actualité au Laboratoire National d'Hydraulique (LNH), compte tenu des applications importantes pour les problèmes d'aménagements littoraux et d'environnement. En particulier, les fines particules sédimentaires d'origine vaseuse, qui présentent des propriétés cohésives, sont d'importants agents de transport de substances tels les micropolluants organiques, noyaux radioactifs, ..., dont une dissémination est préjudiciable à l'équilibre écologique d'un milieu naurel (notamment dans le cas des estuaires).

De nombreux modèles de transport sédimentaires ont été développés dans le passé. Ces modèles sont en général couplés à un modèle hydrodynamique de turbulence plus ou moins sophistiqué (modèle de longueur de mélange, $k-\varepsilon, \ldots$ ). On utilise classiquement une hypothèse de 'scalaire passif' pour calculer la concentration des sédiments en suspension. Les particules sédimentaires sont ainsi supposées suivre l'écoulement instantané dans la direction horizontale; sur la verticale, on considère en général que la vitesse relative moyenne des sédiments en suspension est égale à la vitesse de chute des particules en eau calme, tandis que le coefficient de diffusion des sédiments est proportionnel à la viscosité turbulente.

L'approche classique ne permet donc pas de prendre en compte l'influence des particules sur l'écoulement moyen et turbulent, ainsi que l'effet des collisions. Cette approche est considéré comme valable pour des concentrations volumiques peu importantes $(\mathrm{C}<0.01)$ et pour des sédiments relativement fins, lorsque le temps de réponse des particules est petit devant les temps charactéristiques de l'écoulement moyen et turbulent (cf. Lumley, 1976). L'hypothèse de 'scalaire passif' n'est donc plus vérifiée en zone de proche paroi où les concentrations sont importantes, ainsi que pour des suspensions floculées. 
L'originalité du LNH est d'aborder les mécanismes du transport sédimentaire à l'aide de la théorie diphasique dont l'intérêt principal est la prise en compte de différents termes d'interaction entre les sédiments et l'écoulement. Le modèle, développé dans un contexte d'applications à des écoulements industriels (lits fluidisés, écoulements gaz-solides, ...) (cf. He et Simonin, 1994), a été adapté pour traiter le problèmes de sédiments en suspension dans le cas d'un écoulement uniforme de type couche limite sur fond plat (modèle ID vertical). Les hypothèses et les équations du modèle diphasique 1D vertical sont présentées dans la partie 2 . Le modèle a été validé tout d'abord dans le cas de sédiments non-cohésifs par comparaison avec des mesures expérimentales. Les résultats obtenus, discutés dans la partie 3 , reproduisent de manière satisfaisante l'influence des particules sur l'écoulement turbulent.

Dans le cas des sédiments cohésifs, la taille et la densité des agrégats et donc leur vitesse de chute varie en fonction des propriétés physico-chimiques de la suspension. Il est essentiel de prendre en compte ces processus de floculation qui gouvernent le comportement dynamique de la suspension. Les modifications apportées au modèle pour prendre en compte ces processus de floculation sont présentées dans la partie 4 . Les résultats obtenus sont discutés dans la partie 5 .

\section{Présentation du modèle diphasique}

De manière générale, les équations pour chacune des deux phases, liquide (1) et solide (2), sont obtenues à partir des équations de Navier-Stokes en appliquant un opérateur de moyenne pondérée par la fonction de présence de chacune des phases. Ces équations moyennes font intervenir les taux de présence respectifs de chacune des phases $\left(\alpha_{\mathrm{i}}\right)$ ainsi que leur densité volumique $\left(\rho_{\mathrm{i}}\right)$.

On considère par la suite un écoulement uniforme, dirigé suivant l'axe des $\mathrm{x}$, au dessus d'un fond plat situé en $\mathrm{y}=0$. On s'intéresse à la répartition verticale des différentes variables (vitesses moyennes de l'écoulement et des particules, $U_{1, x}(y)$ et $\left.\mathrm{U}_{2, \mathrm{x}}(\mathrm{y}), \ldots\right)$. Les équations de conservation de la quantité de mouvement suivant $\mathrm{x}$ des phases liquide et solide sont couplées par un terme de frottement entre les phases, qui s'exprime en fonction de la vitesse relative entre les deux phases et d'un coefficient de frottement $F_{D}$. Les contraintes de cisaillement turbulents de la phase liquide sont modélisées à l'aide d'un coefficient de viscosité turbulente $v_{1}^{\mathrm{c}}$, qui est déterminé par un modèle de turbulence à deux équations pour l'énergie cinétique $q_{1}$ et la dissipation $\varepsilon_{1}$. Dans l'équation de quantité de mouvement pour la phase solide, les composantes cinétiques et collisionnelles des contraintes inter-granulaires sont modélisées, par analogie avec les contraintes turbulentes, en utilisant des coefficients de viscosité cinétique $v_{2}^{\text {cin }}$ et collisionnelle $v_{2}^{\text {col }}$, qui s'expriment en fonction de la vitesse d'agitation des particules $q_{2}$ à l'aide d'un modèle semiempirique (e.g. Lun et al., 1984). Ces équations s'écrivent:

$$
\alpha_{1} \rho_{1} \frac{\partial \mathrm{U}_{1, \mathrm{x}}}{\partial \mathrm{t}}=-\alpha_{1} \frac{\mathrm{d} \mathrm{P}_{1}}{\mathrm{dx}}+\alpha_{2} \rho_{1} \mathrm{~F}_{\mathrm{D}}\left(\mathrm{U}_{2, \mathrm{x}}-\mathrm{U}_{1, \mathrm{x}}\right)+\frac{\partial}{\partial \mathrm{y}}\left[\alpha_{1} \rho_{1}\left(v_{1}+v_{1}^{\mathrm{t}}\right) \frac{\partial \mathrm{U}_{1, \mathrm{x}}}{\partial \mathrm{y}}\right]
$$


$\alpha_{2} \rho_{2} \frac{\partial U_{2, \bar{x}}}{\partial t}=-\alpha_{2} \frac{d P_{1}}{d x}-\alpha_{2} \rho_{1} F_{D}\left(U_{2, x}-U_{1, x}\right)+\frac{\partial}{\partial y}\left[\alpha_{2} \rho_{2}\left(v_{2}^{\text {cin }}+v_{2}^{\text {col }}\right) \frac{\partial U_{2, x}}{\partial y}\right]$

L'équation de transport-diffusion pour le taux de présence de la phase solide $\alpha_{2}$ est obtenue à partir des équations de continuité pour chacune des phases. La diffusion turbulente des particules est modélisée à l'aide d'un coefficient de diffusion turbulente $D_{12}^{t}$ qui représente la turbulence du fluide 'vue' par les particules au cours de leur mouvement relatif et qui est fonction de la corrélation entre les vitesses de l'écoulement et des particules $\left(\mathrm{q}_{12}\right)$. Le flux moyen des particules est exprimé en fonction de $\mathrm{V}_{t}$, vitesse relative verticale entre les phases 1 et 2 .

$$
\frac{\partial \alpha_{2} \rho_{2}}{\partial \mathrm{t}}-\frac{\partial}{\partial \mathrm{y}}\left(\mathrm{D}_{12}^{\mathrm{t}} \frac{\partial \alpha_{2} \rho_{2}}{\partial \mathrm{y}}\right)+\frac{\partial}{\partial \mathrm{y}}\left(\alpha_{1} \alpha_{2} p_{2} V_{\mathrm{r}}\right)=0
$$

La vitesse $V_{r}$ est déterminée comme solution du modèle en écrivant l'équilibre vertical entre les forces de frottement, de lift et de pression intergranulaire. Elle differe de la vitesse de chute en eau claire $W_{s}$, notamment en zone de proche paroi. En effet, compte tenu du cisaillement des vitesses et des concentrations importantes près du fond, la force de lift (coefficient $C_{L}$ ) et la pression intergranulaire $\left(\mathrm{P}_{2}\right)$ ont pour effet de réduire de manière significative la vitesse $V_{r}$. On écrit:

$$
\alpha_{1} \alpha_{2} \mathrm{~V}_{\mathrm{r}}=\frac{\alpha_{1}^{2} \alpha_{2}}{\mathrm{~F}_{\mathrm{D}} \mathrm{C}_{\mathrm{y}}} \frac{\left(\rho_{2} \rho_{1}\right)}{\rho_{1}} \mathrm{~g}-\frac{\alpha_{1} \alpha_{2}}{\mathrm{~F}_{\mathrm{D}} \mathrm{C}_{\mathrm{y}}} \mathrm{C}_{\mathrm{L}} \frac{1}{\mathrm{~d}} \sqrt{\nu_{1} \frac{\partial \mathrm{U}_{1, \mathrm{x}}}{\partial \mathrm{y}}}\left(\mathrm{U}_{2, \mathrm{x}}-\mathrm{U}_{1, \mathrm{x}}\right)-\frac{\alpha_{1}^{2}}{\rho_{1} \mathrm{~F}_{\mathrm{D}}} \frac{\partial \mathrm{P}_{2}}{\partial y}
$$

Les équations de transport-diffusion pour les grandeurs turbulentes $q_{1}, \varepsilon 1, q_{12}, q_{2}$ sont analogues aux équations d'un modèle classique de turbulence $k-\varepsilon$ avec prise en compte de termes d'interaction entre la turbulence de l'écoulement et la suspension. Les équations du modèle et les conditions aux limites (vitesse nulle à la paroi) sont discrétisées dans un maillage vertical à progression géométrique qui permet de rafiner la zone en proche paroi. Le système d'équation est discrétisé de manière implicite: on aboutit à la résolution d'un système matriciel: $[\mathrm{A}]\left[\mathrm{X}^{\mathrm{n}+1}\right]=[\mathrm{B}]\left[\mathrm{X}^{\mathrm{n}}\right]+[\mathrm{C}]=[\mathrm{D}]$ avec $[\mathrm{A}]$ une matrice tridiagonale.

\section{Validation du modèle}

Dans un premier temps, le modèle diphasique a été utilisé pour simuler le transport de sédiments non cohésifs, afin de valider les processus d'interaction entre l'écoulement moyen et turbulent et les sédiments en suspension (cf. Villaret et Davies, 1995). On considère les mesures en laboratoire de Lyn (1988) et Coleman (1981). Les paramètres expérimentaux sont indiqués dans le tableau 1.

\begin{tabular}{|l|l|l|l|l|l|}
\cline { 2 - 6 } \multicolumn{1}{c|}{} & $\mathrm{h}(\mathrm{cm})$ & $\mathrm{U}(\mathrm{cm} / \mathrm{s})$ & $\mathrm{d}(\mathrm{mm})$ & $\mathrm{C}\left(\times 10^{3}\right)$ & $\mathrm{Ws}(\mathrm{cm} / \mathrm{s})$ \\
\hline Lyn 65ST & 6,6 & 68.3 & 0,19 & 0,27 & 2,5 \\
\hline Coleman 31 & 17,0 & 95.0 & 0,21 & 1,8 & 3,0 \\
\hline
\end{tabular}

Tableau 1: Paramètres expérimentaux (hauteur d'eau $h$, vitesse moyenne débitante $\mathrm{U}$, diamètre des sédiments $\mathrm{d}$, concentration volumique moyenne $\mathrm{C}_{0}$ et vitesse de chute $W_{s}$ ). 
Les résultats numériques sont comparés avec les mesures des profils verticaux des vitesses et concentrations ( $\mathrm{cf}$. Figures let 2 ). Les résultats sont considérés comme satisfaisants. On constate que, en accord avec les mesures expérimentales, l'influence des particules sur l'écoulement a pour effet d'augmenter la rugosité apparente ainsi que la pente du profil des vitesses moyennes par rapport au profil des vitesses en eau claire (i.e. trait plein).

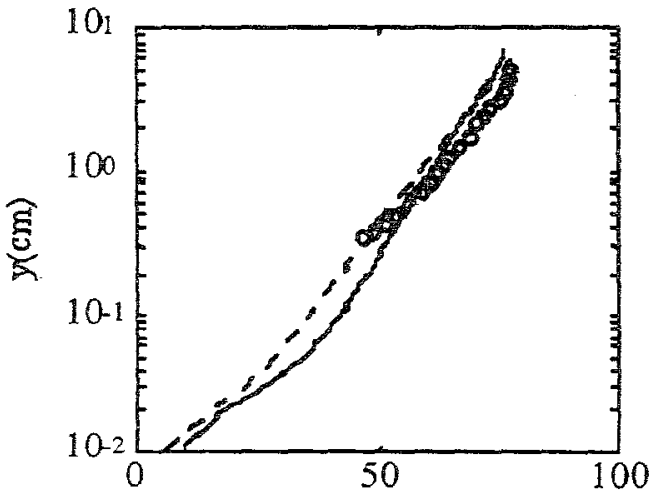

Fig. 1.a Profil de vitesse U1 $(\mathrm{cm} / \mathrm{s})$

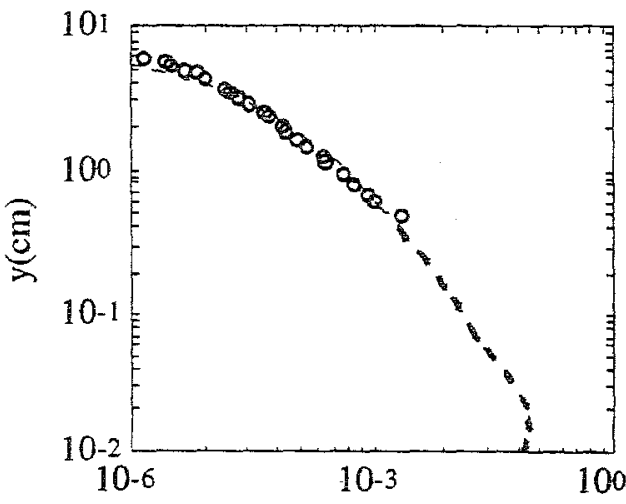

Fig. 1.b: Profil de concentration

Figures 1: Comparaison entre les résultats du modèle diphasique (- -) et les données expérimentales de Lyn(o). Le trait plein correspond au profil des vitesses en eau claire calculé par le modèle.
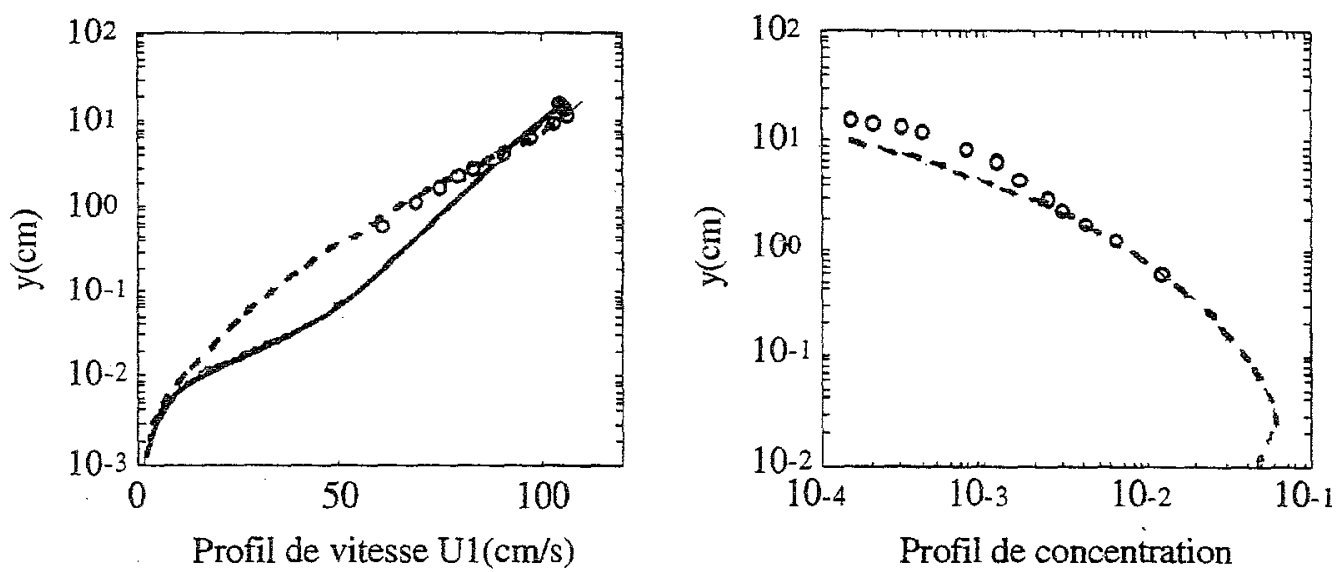

Figures 2: Comparaison avec les données expérimentales de Coleman (o).

\section{Modélisation du transport de sédiments cohésifs}

\subsection{Propriétés cohésives}

Les sédiments cohésifs, constitués de particules élémentaires très fines, de l'ordre de quelques microns ont la propriété de s'agglomérer entre elles pour former des agrégats de dimensions très supérieures $(0.1$ à $2 \mathrm{~mm})$. Lorsque les flocs augmentent en taille, ils se gorgent d'eau et donc leur densité moyenne diminue. D'après des études expérimentales (cf.Van Leussen, 1994 ), la densité des agrégats est inversement proportionnelle à leur diamètre: 
$\rho_{2}=\rho_{1}+\frac{\mathrm{A}}{\mathrm{d}}$

où $A$ est une constante dépendant de la nature des sédiments $\left(A \approx 10^{-3}\right)$.

Les caractéristiques des flocs dépendent des propriétés physico-chimiques de la suspension (salinité, concentration, ...). La turbulence de l'écoulement agit aussi sur la taille des flocs de deux manières opposées: pour de petites particules, une augmentation de la turbulence entraîne une augmentation du nombre de collisions par unité de temps, ce qui fait apparaitre des flocs de plus en plus gros et fragiles. D'autre part, une augmentation de la turbulence entraîne une augmentation des forces de cisaillement et de pression qui vont entraîner la rupture des flocs : la turbulence est donc aussi une limitation de la taille des flocs.

\subsection{Equations du modèle}

Les mécanismes de floculation qui gouvernent le comportement dynamique des sédiments cohésifs, sont ici inclus dans le modèle diphasique. Pour cela, on considère comme variables le diamètre des agrégats $\mathrm{d}(\mathrm{y}, \mathrm{t})$ et leur densité $\rho_{2}(\mathrm{y}, \mathrm{t})$. On résoud une équation supplémentaire de transport-diffusion pour le nombre de particules par unité de masse $X_{p}$ qui est relié au diamètre et à la densité des flocs par la relation:

$\frac{\pi \mathrm{d}^{3}}{6}=\frac{1}{\rho_{2} \mathrm{X}_{\mathrm{p}}}$

$\alpha_{2} \rho_{2} \frac{\partial \mathrm{X}_{\mathrm{p}}}{\partial \mathrm{t}}=\frac{\partial}{\partial \mathrm{y}} \alpha_{2} \rho_{2} \mathrm{D}_{2}^{\mathrm{r}} \frac{\partial \mathrm{X}_{\mathrm{p}}}{\partial \mathrm{y}}-\mathrm{X}_{\mathrm{p}} \Gamma_{2}+\alpha_{2} \rho_{2} \Gamma_{\mathrm{P}}$

Dans cette équation, $\Gamma_{2}$ représente un terme d'échange de masse et $\Gamma_{p}$ un terme de production-destruction du nombre de particules, avec $\Gamma_{\mathrm{P}}>0$ pour la destruction d'agrégats et $\Gamma_{\mathrm{p}}<0$ pour la formation d'agrégats. Le coefficient de dispersion $D_{2}^{t}$ est calculé en fonction du coefficient de diffusion et du temps de réponse des agrégats:

$D_{2}^{\mathrm{t}}=\mathrm{D}_{12}^{\mathrm{t}}+\frac{2}{3} \mathrm{q}_{2}^{2} \tau_{12}^{\mathrm{F}}$ avec $\tau_{12}^{\mathrm{F}}=\frac{\rho_{2}}{\rho_{1} \mathrm{~F}_{\mathrm{D}}}$.

Le terme d'échange de masse $\Gamma_{2}$ doit aussi apparaitre dans l'équation de continuité qui s'écrit:

$\frac{\partial \alpha_{2} \rho_{2}}{\partial \mathrm{t}}-\frac{\partial}{\partial \mathrm{y}}\left(D_{12}^{\mathrm{t}} \frac{\partial \alpha_{2} \rho_{2}}{\partial \mathrm{y}}\right)+\frac{\partial}{\partial \mathrm{y}}\left(\alpha_{1} \alpha_{2} \rho_{2} \mathrm{~V}_{\mathrm{r}}\right)=\Gamma_{2}$

\subsection{Expression de $\Gamma_{P}$.}

- Pour la formation des agrégats $\left(\Gamma_{\mathrm{P}}<0\right)$, le facteur principal de collisions est le cisaillement turbulent $\mathrm{G}$, défini par: 


$$
\mathrm{G}=\sqrt{\frac{\varepsilon_{1}}{v_{1}}}
$$

On introduit aussi un facteur d'efficacité des collisions $\gamma<1$ qui représente la probabilité pour que des particules qui rentrent en contact forment un agrégat ( $\gamma=$ 0.03 correspond à des sédiments peu cohésifs et $\gamma=0.2$ à des sédiments hautements cohésifs). Ce facteur dépend de la nature des sédiments (diamètre) ainsi que des propriétés physico-chimiques du milieu acqueux (salinité). Le terme de production d'agrégat $\left(\Gamma_{\mathrm{P}}<0\right)$ s'exprime alors (cf.Van Leussen, 1994):

$$
\left.\Gamma_{\mathrm{P}}=\frac{\partial \mathrm{X}_{\mathrm{P}}}{\partial \mathrm{t}}\right)_{\text {formation }}=-\frac{4}{\pi} \gamma \alpha_{2} \mathrm{G} \mathrm{X}_{\mathrm{P}}
$$

- Pour le processus de destruction des agrégats $\left(\Gamma_{P}>0\right)$, l'expression de $\Gamma_{P}$ est méconnue. $D$ 'après les mesures expérimentales, le diamètre des flocs atteint une valeur maximale $d_{\max }$, définie comme la taille limite du floc pouvant résister aux forces hydrodynamiques sans être disloqué.

On choisit de modéliser $\Gamma_{\mathrm{P}}$ de la manière suivante : $\left.\Gamma_{\mathrm{P}}\right)_{\text {destruction. }}=\frac{18}{\rho_{2} \pi} \mathrm{d}^{-4} \frac{\left(\mathrm{d}-\mathrm{d}_{\max }\right)}{\Delta \mathrm{t}}$

- Expression du diamètre maximum $\mathrm{d}_{\max }$

En l'absence de données expérimentales précises, on peut supposer par une analyse dimensionnelles que le diamètre des agrégats est de l'ordre de la micro-échelle de Kolmogorov:

$\mathrm{d}_{\max } \eta$, avec $\eta=\left(\frac{v_{1}^{3}}{\varepsilon_{1}}\right)^{\frac{1}{4}}$

\subsection{Modélisation de $\Gamma_{2}$}

La présence de l'eau piégée dans les flocs nécessite la prise en compte de termes d'échanges de masse entre les particules et le fluide.

$\Gamma_{2}=\frac{\partial}{\partial \mathrm{t}}\left(\alpha_{2} \rho_{2}\right)$, soit $\Gamma_{2}=\left(\alpha_{2} \frac{\partial \rho_{2}}{\partial \mathrm{d}}+\rho_{2} \frac{\partial \alpha_{2}}{\partial \mathrm{d}}\right) \frac{\partial \mathrm{d}}{\partial \mathrm{t}}$.

En utilisant la relation empirique $\rho_{2}$ (d) et en considérant comme constant le nombre de particules par unité de volume, on trouve:

$\Gamma_{2}=\left(-\alpha_{2} \frac{\mathrm{A}}{\mathrm{d}^{2}}+\frac{3 \alpha_{2} \rho_{2}}{\mathrm{~d}}\right) \frac{\Delta \mathrm{d}}{\Delta \mathrm{t}}$.

\subsection{Mise en oe uvre numérique:}

Les mécanismes de collisions (formation-destruction) et de changement de densité par absorption d'eau sont découplés en utilisant une méthode de pas fractionnaires de la manière suivante: 


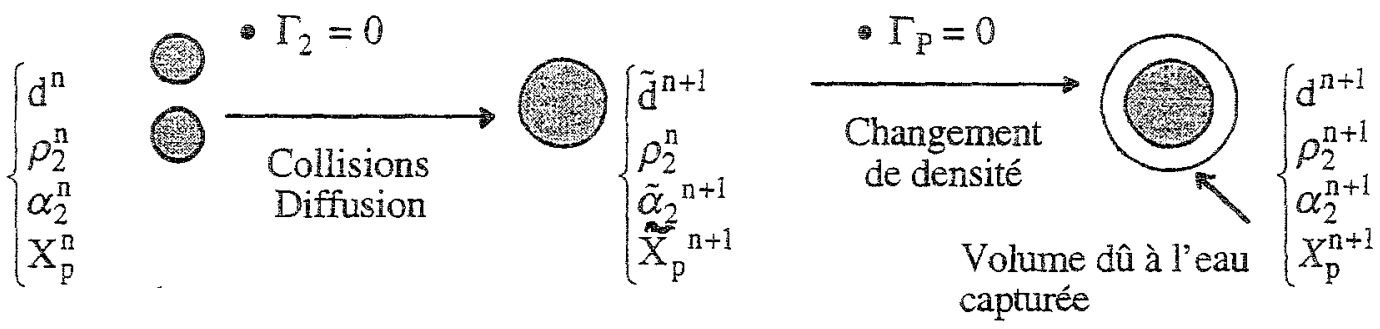

La première étape, la densité des agrégats est considérée comme constante $\left(\Gamma_{2}=0\right)$. Le changement de volume des agrégats est due à une variation de leur nombre sous l'effet du cisaillement turbulent. Pendant la deuxième étape, le nombre de particules par unité de volume est constant $\left(\Gamma_{P}=0\right)$. La densité des agrégats varie par le terme d'échange de masse.

\section{Simulation numérique des mécanismes de floculation}

Le modèle diphasique est ici appliqué au transport de sédiments cohésifs dans un écoulement cisaillé. Il n'existe pas de mesures détaillées, notament en ce qui concerne la structure des agrégats, pour permettre de valider les processus de floculation. Les résultats obtenus servent à illustrer les phénomènes et seront comparés de manière qualitative avec les observations disponibles.

\subsection{Floculation sans changement de densité}

Les conditions hydrodynamiques correspondent à une hauteur d'eau $h=1 \mathrm{~m}$ et une vitesse moyenne débitante de $50 \mathrm{~cm} / \mathrm{s}$. Le profil vertical des vitesses en eau claire correspond à un profil logarithmique.

A l'instant $t=0$, on démarre la simulation avec des particules élémentaires déflocculées ( $d=5 \mu$ et $\rho_{2}=2650 \mathrm{~kg} / \mathrm{m}^{3}$ ). La concentration est uniforme à $4 \mathrm{~g} / \mathrm{l}$. Le diamètre maximal est imposé de manière arbitraire par une loi en $\mathrm{d}_{\max }=\frac{\eta}{5}$. Les résultats des simulations mettent en évidence les différentes phases suivantes:

$-t=0-1 h$ (fig. 3.a): les agrégats sont en formation sur toute la hauteur d'eau. Le diamètre des agrégats a atteint plus de $15 \mu$ au fond.

- $t=1-4 h$ (fig. $3 . b-3 . c$ ): la destruction des agrégats est localisée à quelques points près du fond, tandis que les agrégats continuent à croître sur la majeure partie de la colonne d'eau

- $>$ 4h (fig.3.d): le diamètre n'évolue pratiquement plus. La destruction localisée près du fond entraîne une limitation du diamètre sur toute la colonne d'eau. 

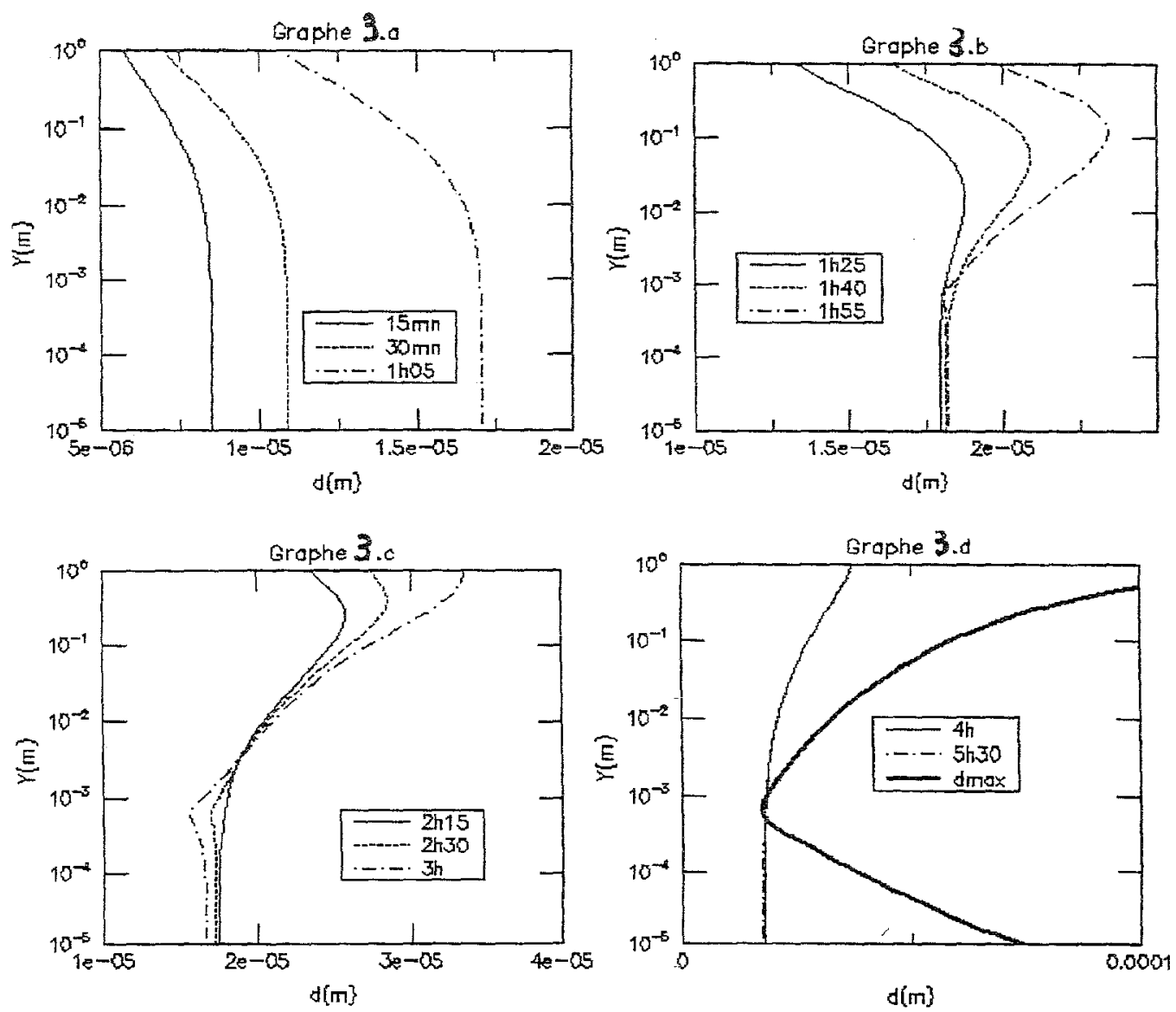

Figure 3: Floculation à densité constants $\left(\rho_{2}=2650 \mathrm{~kg} / \mathrm{m}^{3}\right)$. Evolution du profil vertical des diamètres à partir d'un état défloculé $(\mathrm{d}=5 \mu)$.

Des études de sensibilité sur le diamètre $d_{\max }$ ont par ailleurs été effectuées en utilisant des lois en $\mathrm{A} / \mathrm{G}$ ou $\mathrm{A} \eta$. Le choix du diamètre dmax a une influence déterminante sur les charactéristiques du profil d'équilibre.

\subsection{Floculation avec changement de densité}

La prise en compte du terme d'échange de masse permet d'accélerer les mécanismes de floculation. Au bout d'environ $6 \mathrm{mn}$, le diamètre atteint est 10 fois plus important que celui obtenu à densité constante au bout de $t=1 \mathrm{~h} 25 \mathrm{mn}$ (cf figures 4 ). L'évolution du diamètre au cours du temps est exponentielle: plus la taille des flocs augmente au cours du temps, plus ils capturent de l'eau en floculant. 

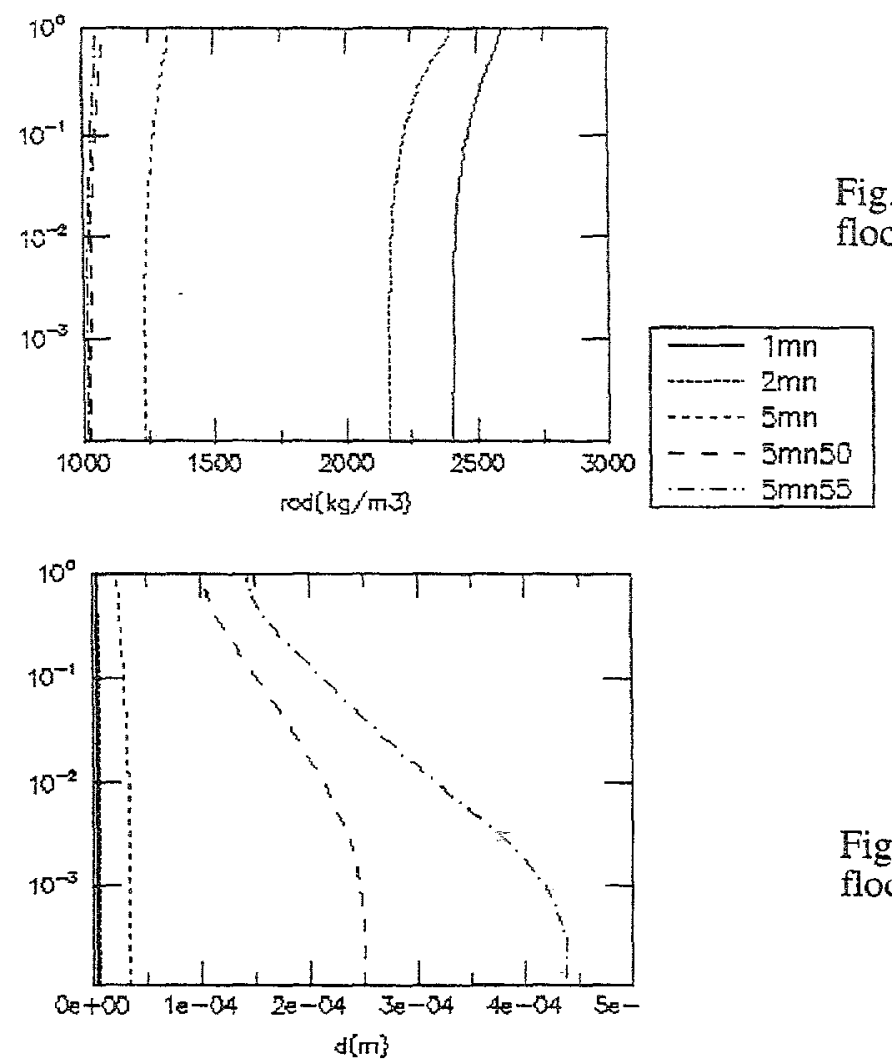

Fig. 4.a: Evolution de la densité des flocs

Fig. 4.b: Evolution du diamètre des flocs

Pour atteindre un profil d'équilibre, on impose un diamètre $d_{\max }=\eta / 5$ comme dans le cas précédent. L'évolution très rapide du diamètre permet d'obtenir un profil d'équilibre en moins de $6 \mathrm{mn}$ (cf fig.5).

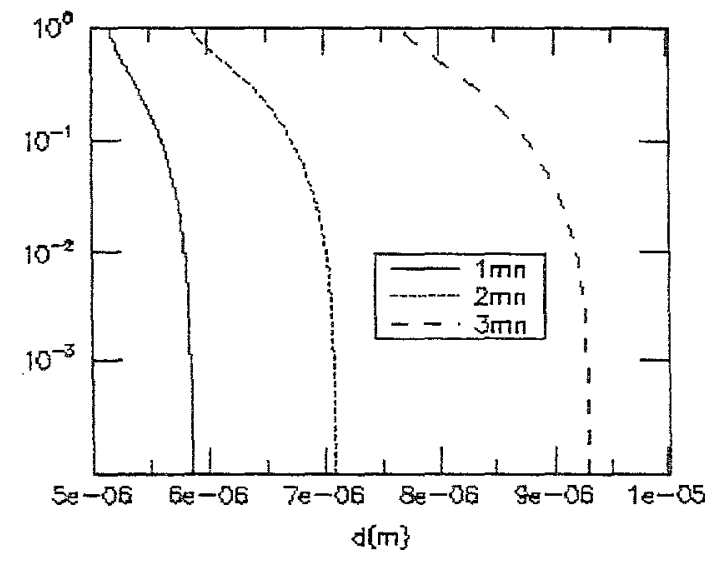

Fig. 5.a: Evolution du diamètre des flocs;

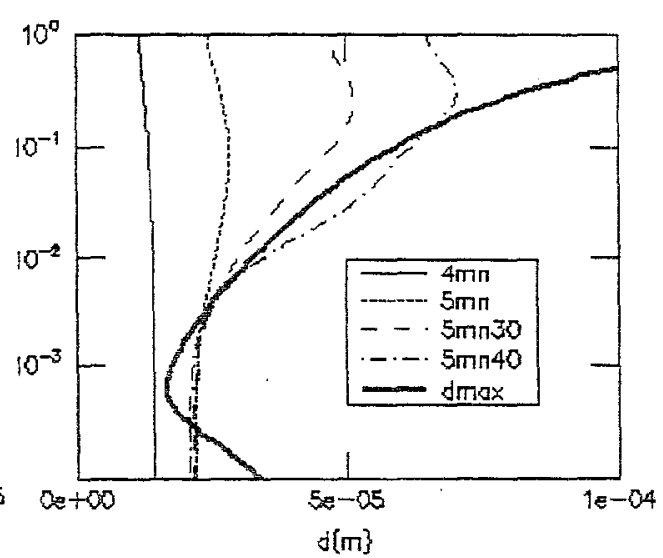

Fig. 5.b: Profil d'équilibre du diamètre

\section{Conclusions}

Le modèle diphasique développé au $\mathrm{LNH}$ a été appliqué au problème du transport de sédiments en suspension dans un écoulement $1 \mathrm{D}$ vertical sur fond plat. 
Le modèle a tout d'abord été validé dans le cas de sédiments non-cohésifs. La comparaison des profils de vitesse et de concentration avec des mesures disponibles est satisfaisante et montre que les interactions entre la turbulence de l'écoulement et les particules sont correctement prises en compte par le modèle.

Le modèle a été ensuite adapté pour prendre en compte les mécanismes de floculation qui régissent le transport des particules cohésives. Les résultats obtenus semblent globalement réalistes. Cependant certains paramètres du modèle (notament le diamètre maximum) ont une influence déterminante sur les résultats obtenus.

\section{REFERENCES}

Coleman NL, Velocity profiles with suspended sediment, Journal of Hydraulic Research, 19, 211-229, 1981.

He J, Simonin O., Modélisation numérique des écoulements gaz-solides en conduite verticale, Rapport EDF, HE-44/94/021A, 1994.

Lyn DA, A similarity approach to turbulent sediment-laden flows in open channels, Journal of Fluid Mechanics, 193, 1-26, 1988.

Lumley JL, Two-phase and non-Newtonian flows, Turbulence, P.Bradshaw (ed.), Springer-Verlag, Berlin, 290-324, 1976.

Lun, C.K.K., Savage, S.B., Jeffery, D.J., Chepurmy, N., Kinetic Theories for Granular Flows : Inelastic Particle in a General Flowfield J. Fluid Mech., Vol 140, pp.223-256, 1984.

Van Leussen, W., Estuarine Macroflocs and their role in fine-grained sediment transport, Thèse de doctorat, Universiteit Utrecht, Nederlands, 1994.

Villaret,C., Davies, A.G., Modelling sediment-turbulent flow interactions, Applied Mechanics Review, vol.48, N9; pp.601-609, 1995. 